\title{
Jenseits von Managed Care
}

\author{
Das Referendum «Für eine freie Arztwahl» und gegen die Managed-Care-Vorlage des \\ Parlamentes ist zustande gekommen. Mit diesem Referendum wird die Chance ge- \\ schaffen, eine über Jahre festgefahrene und zunehmend «kranke» Dynamik im letzten \\ Moment zu stoppen und in eine neue Richtung zu lenken. Woran ist das bisherige \\ Versicherungssystem erkrankt, wie könnte ein Heilungsprozess aussehen? Und wes- \\ halb wird das Referendum allein noch keinen nachhaltigen Systemwechsel bewirken?
}

\section{Michael Sonntag}

Facharzt für Psychiatrie und Psychotherapie, Inhaber eines Unternehmens für Beratungen im Gesundheitswesen
Eine ausführlichere Version inklusive ausführlicher Literaturangaben steht unter www.sonntag-consulting.ch/ sites_de/download.html zur Verfügung.

Korrespondenz:

Dr. med. Michael Sonntag

Manuelstrasse 71

CH-3006 Bern

Tel. 0313528720

m.sonntag[at]

sonntag-consulting.ch
Ein vertrautes Bild: Der Blutdruck des «Patienten Gesundheitswesen» fällt ständig, trotz immer höherer Dosen vermeintlich altbewährter Medikamente und neuer gesundheitspolitischer «Wundermittel». Höchste Zeit also, sich einmal Gedanken über die gestellte Diagnose und die angewandten therapeutischen Massnahmen zu machen. Noch wichtiger ist aber, dass wir uns grundsätzlich bewusst machen, von welchem Menschenbild wir ausgehen. Dieses bestimmt nämlich unser Denken, unser Handeln und die angewandten ökonomischen und Managementmodelle.

\section{Welche Menschenbilder liegen unseren Handlungen zugrunde?}

Die Menschenbilder, an denen sich - oft unbewusst auch die Akteure des Gesundheitswesens orientieren, lassen sich in Anlehnung an D. McGregors Buch The Human Side of Enterprise [1] klassischerweise als folgende zwei Grundtypen vereinfachend beschreiben:

Typ X: unselbständig, uneinsichtig, egoistisch, reiner Kostenfaktor und somit zu kontrollieren und notfalls zu bestrafen. Stichworte: Taylorismus, Command and control, (angebliche) Effizienz, Managed Care mit Budgetverantwortung, Erhöhung des Selbstbehaltes bei freier Arztwahl, DRG, Zentralisierung, Einheitskasse, Organisations- und Managementmodelle aus der Zeit der Industrialisierung und immer mehr «Produktion von wertlosem Unsinn» (M. Binswanger, Sinnlose Wettbewerbe [2]).

Typ Y (oder Typ I nach Daniel H. Pink, [3]): mitdenkende, durch intrinsische Motivation, persönliche Werte und Haltungen (purpose) geleitete, selbstbestimmte, selbstverantwortlich und autonom handelnde Menschen. Stichworte: Engagement, Selbstverantwortung, Selbstbestimmung, freie Arztwahl, Verzicht auf Mikromanagement (Kontrolle); dezentrale, selbstorganisierende, selbstregulierende, vollständig auf Kunden und Mitarbeiter ausgerichtete, wertegesteuerte Organisations- und Managementmodelle; Beyond-Budgeting-Managementmodell.
Auch wäre es hilfreich, wenn wir uns wieder auf die Voraussetzungen besinnen würden, die für gesundes menschliches «Funktionieren» unabdingbar sind: verlässliche, vertrauensvolle, emotional tragende, nahe Beziehungen. Stichworte dazu sind: freie Arztwahl mit gutentwickelter, auf gegenseitigem Vertrauen aufbauender Arzt-Patienten-Beziehung.

\section{Weg vom Budgetdenken}

Solange wir uns nicht von dem veralteten Typ-XMenschenbild und der damit verbundenen Command-and-Control-Kultur verabschieden, wird keine Veränderung stattfinden - und der Blutdruck unseres «Patienten» wird weiter sinken, wohl bis zum «Multiorganversagen» und Zusammenbruch des bisherigen Systems. Der Schritt, sich vollständig vom klassischen Budgetdenken, also der Kontrolle über Geld, zu lösen, ist allerdings sehr schwierig. Unmöglich ist er aber keineswegs. Beispiele moderner Firmen, die eine radikale Transformation in die skizzierte Richtung gemacht haben, gibt es reichlich auch im Gesundheitswesen.

So stockte die Group Health Cooperative, ein grosser Gesundheitsversorger in Seattle, das ambulant tätige ärztliche Personal um rund $60 \%$ auf. Mehrkosten: 16 Dollar pro Patient und Jahr. Einsparungen binnen eines Jahres: 54 Dollar pro Patient, allein durch 30\% weniger Notfalleinweisungen [4].

Die Park-Nicolett-Klinikgruppe in Minneapolis setzte bereits im Jahr 2005 ein konsequent patientenzentriertes Modell binnen eines Jahres erfolgreich um. Hierfür wurden innere Service-Linien über die Abteilungen hinweg geschaffen. Der klassische Budgetierungsprozess wurde komplett eliminiert, die Entscheidungskompetenz an den Ort des Patientenkontaktes dezentralisiert. Neben massiven Kosteneinsparungen und einer Verbesserung der Qualität resultierte eine eindrückliche Verbesserung, nicht nur der Patienten-, sondern auch der Mitarbeiterzufriedenheit [5]. 


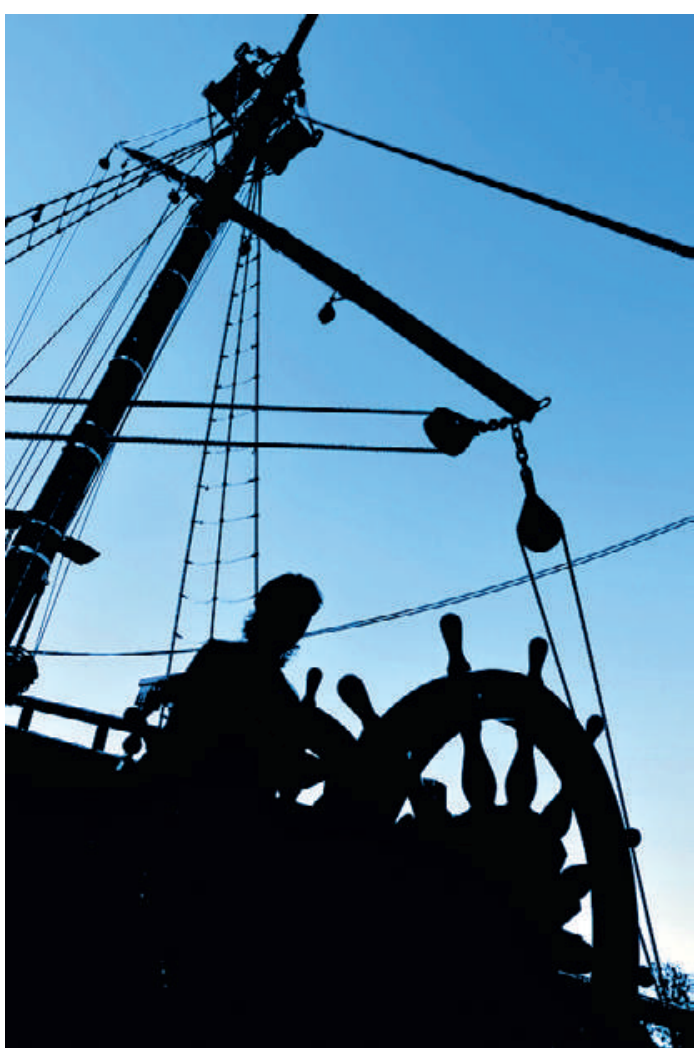

Können die Ärzte durch das Managed-Care-Referendum das Ruder wieder selber in die Hand und Kurs nehmen auf ein patientenzentriertes und wertgenerierendes Gesundheitswesen?

\section{Gewinnchance oder Systemkollaps?}

Es braucht aber den Mut zu einem radikal neuen Verständnis von Wert-Generierung und das Entwickeln entsprechender Organisations- und Managementmodelle. Eine reine Optimierung von tradierten Steuerungsinstrumenten wird nicht reichen.

Die freie Arztwahl ist hier ein wichtiger Schritt in die richtige Richtung, wird aber noch keinen Systemwechsel bewirken. Hierzu braucht es eine Neuorientierung, die den Patienten und seine individuelle medizinische, psychische und soziale Situation jenseits von kurzfristigem Kostendenken radikal ins Zentrum der Überlegungen und Handlungen stellt. Dies wiederum setzt Organisations- und Managementformen, die ein solches Handeln ermöglichen und unterstützen, voraus [6]. Was hier aber nach aufwendiger Reorganisation klingt, ist im konkreten Fall erschütternd einfach: Es braucht Ärztinnen und Ärzte, die sich unlimitiert die nötige - gutbezahlte Zeit nehmen können, um zusammen mit dem Patienten und den mitbehandelnden Kolleginnen und Kollegen die in seiner aktuellen, individuellen Situation richtigen medizinischen Entscheide zu fällen unabhängig von Kosten. Und siehe da, plötzlich wird alles viel einfacher und billiger. Dazu braucht es also nicht komplexe Kontroll- und Steuerungsinstrumente, sondern ein Managementmodell, das radikal von selbstbestimmten, verantwortungsbewussten und mündigen Ärzten und Patienten ausgeht. Ein soeben im Harvard Business Review publizierter Artikel zeigt, wie auf diese Weise riesige Summen gespart werden können, aber nur wenn wirkliches Vertrauen besteht und die Entscheide nicht primär zum Kostensparen gefällt wurden [7]. Unlogisch? Nein, logisch! Ineffizient? Nein, hocheffizient!

Die aktuelle Managed-Care-Vorlage des Parlaments ist immer noch ein Modell, das auf dem alten Command-and-controll-Prinzip aus dem letzten Jahrhundert basiert. Das Ziel der Vorlage ist nicht das Generieren von value, sondern die Kontrolle über Geld. Erfolgreiche, agile, innovative Firmen haben diese alten Prinzipien längst hinter sich gelassen, während unsere Gesundheitspolitiker versuchen, ein längst überholtes Modell, das ökonomisch komplett versagt hat, für die nächsten Jahrzehnte zu zementieren.

Diejenigen Akteure im Gesundheitswesen (insbesondere die Krankenkassen), die es wagen würden, entsprechende «Beyond-Managed-Care»-Projekte interdisziplinär umzusetzen, könnten viel Geld verdienen - durch verringerte Kosten, Gewinnen vieler neuer (Typ I)-Kunden und durch die Kooperation mit wirklich guten, nachhaltig denkenden und handelnden Ärzten.

Das Referendum bietet somit nicht nur die eventuell letzte Chance, das Ruder herumzureissen, sondern es könnte auch die Gelegenheit bieten, das Ruder selber in die Hand zu nehmen und den Kurs des Schiffes in Richtung eines modernen, patientenzentrierten, wertgenerierenden Gesundheitswesens zu lenken.

\section{Literatur}

1 McGregor D. The Human Side of Enterprise. McGrawHill: 1960. Annotated edn. McGraw-Hill; 2006.

2 Binswanger M. Sinnlose Wettbewerbe - Warum wir immer mehr Unsinn produzieren. Freiburg i. Br.: Herder; 2010.

3 Pink DH. Drive - The surprising truth about what motivates us. Canongate Books; 2010.

4 Reid RJ. Patient-Centered Medical Home Demonstration: A Prospective, Quasi-Experimental, Before and After Evaluation. Am J Manag Care. 2009;15(9):e71-87.

Oder: www.grouphealthresearch.org/news-andevents/newsrel/2009/090901.html

5 McVay GJ, Cooke DJ. Beyond Budgeting in an IDS: The Park Nicollet Experience. Healthcare Financial Management Association hfma. 2006;10.

6 Röösli F, Sonntag M. Mehr Potential dank Vernetzung Ein Plädoyer für Management-Plastizität: Warum Organisationen im Informationszeitalter auf vernetzte und lernfähige Strukturen setzen sollten. io Management. 2011; November/Dezember:34-37.

Oder: www.iomanagement.ch/de/artikelanzeige/ artikelanzeige.asp?pkBericht $\mathrm{Nr}=184146$

7 Goodman E. Die the Way You Want To. Harvard Business Review. 2012; January/February. Oder: http://hbr.org/2012/01/tackling-socialproblems/ar/1 